\title{
Stochastic resonance in periodically driven bistable systems subjected to anomalous diffusion
}

\author{
F. Naha Nzoupe ${ }^{1} \cdot$ Alain M. Dikandé ${ }^{1}$
}

Received: 3 November 2020 / Accepted: 23 February 2021 / Published online: 6 March 2021

(C) The Author(s) 2021 OPEN

\begin{abstract}
The occurrence of stochastic resonance in bistable systems undergoing anomalous diffusions, which arise from densitydependent fluctuations, is investigated with an emphasis on the analytical formulation of the problem as well as a possible analytical derivation of key quantifiers of stochastic resonance. The nonlinear Fokker-Planck equation describing the system dynamics, together with the corresponding Ito-Langevin equation, is formulated. In the linear response regime, analytical expressions of the spectral amplification, of the signal-to-noise ratio and of the hysteresis loop area are derived as quantifiers of stochastic resonance. These quantifiers are found to be strongly dependent on the parameters controlling the type of diffusion; in particular, the peak characterizing the signal-to-noise ratio occurs only in close ranges of parameters. Results introduce the relevant information that, taking into consideration the interactions of anomalous diffusive systems with a periodic signal, can provide a better understanding of the physics of stochastic resonance in bistable systems driven by periodic forces.
\end{abstract}

Keywords Bistable systems · Anomalous diffusion · Periodic forcing $\cdot$ Stochastic resonance $\cdot$ Signal-to-noise ratio $\cdot$ ItoLangevin equation

\section{Introduction}

Nonlinear systems exhibit a broad range of unexpected behaviors as well as complex dynamical properties, due to the presence of noise [1-5]. As typical example, thermally activated processes such as noise-induced escape from metastable states [1-5] and the phenomenon of stochastic resonance $(\mathrm{SR})[3,6,7]$ have attracted a great deal of attention because of their fundamental role in several areas of physics, chemistry, biophysics, social as well as financial sciences [5, 8-10]. In particular, there has been an unprecedented interest in noise-driven phenomena pertaining to the so-called Kramers escape rate problem [5], due to their intimate connection with second-order phase transitions [11] in physical contexts where dynamical instabilities are governed by double-well potentials [12-14]. For these systems, noise-driven transport phenomena are well known to be dominated by standard diffusion processes, whereby oscillators exhibit normal Brownian motions and the escape rate has the standard Arrhenius law [3]. Investigations of SR in systems of this class have led to the important conclusion that noise multiplicativity and time correlation are necessary for the occurrence of SR in linear systems, and that nonlinearity is an essential ingredient for the occurrence of SR in physical contexts with additive white noise [15-19]. Also required is the interplay of at least two possible equilibrium states with sufficiently confining strength and separated by a potential barrier [20-22].

In nature, we equally encounter physical systems in which noise-driven transports involve diffusion phenomena that deviate strongly from the classic picture. Indeed

Alain M. Dikandé, dikande.alain@ubuea.cm | 'Laboratory of Research on Advanced Materials and Nonlinear Science (LaRAMaNS), Department of Physics, Faculty of Science, University of Buea, P.O. Box 63, Buea, Cameroon. 
certain diffusion phenomena exhibit nonlinear transport features which are completely distinct from the standard Brownian motion, resulting in an anomalous behavior of the escape dynamics [23-27]. Moreover, they display different scenarios of occurrence of SR, as evidenced by some recent results on this issue [23-25].

In this work, we are interested in SR for systems exhibiting correlated-type anomalous diffusions; in particular, we focus on physical contexts where diffusion processes depend on the probability density $P$ of particles. Here, we treat the case where the diffusion coefficient is proportional to an arbitrary power of $P$, such that depending on values of two power coefficients $v$ and $\mu$ the system is either subdiffusive $[26,28]$ or superdiffusive $[26,28]$. Ranging from cell dynamics [29], surface growth [30], radiative heat transfer by Marshak waves [31], gravitational spreading of thin liquid films [32], spatial diffusion of biological populations [33] and saturation of thin regions in porous media [34], there exist a wealth of real physical situations where stochastic processes display anomalous diffusions with a density-dependent diffusion coefficient. Some expressions for the escape time were proposed in the literature $[35,36]$ and were shown to be strongly dependent on the order of anomaly of the system diffusion. To our knowledge, no theoretical investigation of the occurrence of SR in such systems has been performed. It would therefore be particularly interesting to know how systems exhibiting density-dependent diffusion coefficients would respond to an external periodic forcing. First of all, we shall discuss the Kramers escape problem for bistable systems with anomalous diffusion, ignoring the periodic forcing. Then we examine the effects of a sinusoidal-type periodic driving force on the occurrence of SR in the system.

\section{Model and escape rate in the presence of anomalous diffusion}

The class of systems we are interested in can be represented as set of particles immersed in a thermal bath. In order to probe the escape behavior, and the possible occurrence of SR in the systems, we introduce a bistable potential coupled to a periodic forcing. At the macroscopic scale, the system dynamics can be described by the following nonlinear Fokker-Planck equation [26, 27]:

$\partial_{t} P^{\mu}(x, t)=-\partial_{x}\left[f(x, t) P^{\mu}(x, t)\right]+D \partial_{x}^{2} P^{v}(x, t)$,

where $f(x, t)=-\partial_{x} U(x)+A(t)$ is the external force with $U$ a bistable potential of the form $U(x)=U_{0}\left(x^{2}-1\right)^{2}$ and $A(t)$ a sinusoidal periodic forcing, i.e., $A(t)=A_{0} \sin \omega t$. $U_{0}$ and $A_{0}$ are the potential barrier and the magnitude of the periodic force, respectively. The parameter $D$ in Eq. (1) measures the anomalous diffusion-induced noise strength, while $(\mu, v)$ are real and positive parameters characterizing the deviation of the diffusion process from the normal diffusion.

Equation (1) describes a driven bistable system undergoing diffusion processes, which can be either a superdiffusion when $\mu>v$, a normal diffusion when $\mu=v$ or a subdiffusion when $\mu<v[25,26]$. Instructively the double-well potential $U(x)$ has its stable states located at $x_{ \pm}= \pm 1$, with a barrier $E_{b}=U_{0}$ centered at $x_{b}=0$. We first examine the Kramers escape problem in the absence of periodic driving. When $A_{0}=0$, the system dynamics is mainly governed by random fluctuations and may lead to a transition between the states $x_{ \pm}= \pm 1$. The Fokker-Planck equation (1) in this case reduces to:

$\partial_{t} P^{\mu}(x, t)=\partial_{x}\left[P^{\mu}(x, t) \frac{\mathrm{d} U(x)}{\mathrm{d} x}\right]+D \partial_{x}^{2} P^{v}(x, t)$.

By optimizing the entropy of the system [37], the stationary solution to Eq. (2) is straightforward yielding:

$P_{s t}(x)=\frac{1}{Z}\left[1-\left(\frac{v-\mu}{\mu}\right) \beta_{\mu \nu} U(x)\right]_{+}^{\frac{\mu}{v-\mu}}$,

where $[.]_{+}=\max \{., 0\}, \beta_{\mu \nu}$ defined by:

$\beta_{\mu \nu}=\frac{\mu}{v D} Z^{\frac{v-\mu}{\mu}}$,

and the normalization constant $Z=\int\left[1-\mu^{-1}(\nu-\mu) \beta_{\mu \nu} U(x)\right]_{+}^{\frac{\nu}{\nu-\mu}}$. For a nonlinear medium with arbitrary diffusion characterized by values of $(\mu, v)$, it is useful $[35,38]$ to define a suitable $(\mu, v)$-dependent effective potential, which in the present context reads:

$\phi_{e}(x)=\frac{v}{\mu-v} \ln \left[1-\left(\frac{v-\mu}{\mu}\right) \beta_{\mu \nu} U(x)\right]$.

In terms of this effective potential, the exact expression for the current of particles across the effective potential barrier, i.e., $S$, will be:

$S(x, t)=-D \exp \left[-\frac{\phi_{e}(x)}{D}\right] \frac{\partial}{\partial x}\left[\exp \left(\frac{\phi_{e}(x)}{D} P^{v / \mu}(x, t)\right)\right]$.

By invoking Kramers rate theory [3] we obtain the escape rate in the small noise limit, i.e., 


$$
\begin{aligned}
r_{k} \approx & \frac{Z^{\frac{v-\mu}{\mu}} \sqrt{U^{\prime \prime}\left(x_{ \pm}\right)\left|U^{\prime \prime}\left(x_{b}\right)\right|}}{2 \pi} \\
& \times\left[1-\left(\frac{v-\mu}{\mu}\right) \beta_{\mu \nu} U\left(x_{b}\right)\right]_{+}^{\frac{\mu+\nu}{2(v-\mu)}} \\
& \times\left[1-\left(\frac{v-\mu}{\mu}\right) \beta_{\mu \nu} U\left(x_{ \pm}\right)\right]_{+}^{\frac{\mu-3 v}{2(v-\mu)}},
\end{aligned}
$$

where $U^{\prime \prime}=d^{2} U / d x^{2}$. For the double-well potential $U(x)$ defined above, Eq. (7) simplifies to:

$r_{k} \approx \frac{2 \sqrt{2} E_{b} Z^{\frac{\nu-\mu}{\mu}}}{\pi}\left[1-\left(\frac{v-\mu}{\mu}\right) \beta_{\mu \nu} E_{b}\right]_{+}^{\frac{\mu+\nu}{2(v-\mu)}}$.

This is the Kramers escape rate for a bistable system subjected to anomalous diffusion.

Let us now examine the system dynamics taking into consideration the periodic forcing. At the microscopic scale, where the distributions of particle positions in time is meaningful, we need to consider the Ito-Langevin equation [39-41] coupled to Eq. (1), which in the present specific context is expressed:

$\frac{\partial x}{\partial t}=-f(x, t)+g(x, t) \eta(t)$,

with $\eta(t)$ standing for thermal fluctuations. From Eq. (1), the probability distribution of a particle position $x$ at time $t+\Delta t$ must satisfy [5]:

$P^{\mu}(x, t+\Delta t)=\int p\left(x, t+\Delta t \mid x^{\prime}, t\right) P^{\mu}\left(x^{\prime}, t\right) \mathrm{d} x^{\prime}$,

where $p$ denotes the transition probability. Setting $x=x^{\prime}+\Delta x$, a Taylor expansion of the integrand in Eq. (10) for small $\Delta x$ leads to the relation:

$\left\langle\Delta x^{n}\right\rangle=\int \Delta x^{n} p(x+\Delta x, t+\Delta t \mid x, t) \mathrm{d} \Delta x$,

from which the Kramers-Moyal expansion coefficient can be derived [42]. Moreover, based on Eq. (11) it was established that to have a correspondence between Eqs. (1) and (9), with $\eta(t)$ obeying $\langle\eta(t)\rangle=0$ and $\langle\eta(t)\rangle\langle\eta(s)\rangle=\delta(t-s)$, the following relation should hold:

$\frac{1}{2} g^{2}(x, t)=D[P(x, t)]^{\nu-\mu}$.

Using this constraint, we can rewrite the Ito-Langevin equation describing the system dynamics at the microscopic scale as:

$\frac{\mathrm{d} x}{\mathrm{~d} t}=-\frac{\partial U(x)}{\partial x}+A_{0} \sin \omega t+\sqrt{2 D}[P(x, t)]^{\frac{v-\mu}{2}} \eta(t)$.
In the case of normal diffusion (i.e., $\mu=v$ ), Eq. (14) reduces to the Langevin equation for a constant noise. The state dependence of noise in Eq. (14) can be interpreted as a consequence of interactions with environment that cannot be explicitly taken into account by the equations of motion. Equation (14) thus provides a phenomenological description in which the macroscopic quantity $P$ stands for a kind of statistical feedback determining microscopic trajectories for the case of anomalous diffusion $(\mu \neq v)$. During this process, the interactions with the environment are such that as the system evolves, the particle is affected by the surrounding collective density of states.

\section{Stochastic resonance in the presence of periodic driving}

We set the system in the small noise regime and weak modulation strength, i.e., $\left|A_{0} x_{s}\right| \ll E_{b}$ and $\omega \ll U^{\prime \prime}\left(x_{ \pm}\right)$, such that there are no possible transitions between stable states in the absence of noise. Ignoring intrawell dynamics in the Ito-Langevin equation (13), the system response in the long-time regime is governed by its harmonic component, i.e. [3],

$\langle x(t)\rangle=\bar{x}(D) \sin (\omega t-\bar{\phi}(D))$,

where the amplitude $\bar{x}$ and phase lag $\bar{\phi}$, in the linear response approximation, are given by:

$\bar{x}(D)=\left(\frac{3 v-\mu}{2 \mu}\right) \frac{2 A_{0} \beta_{\mu \nu}\left\langle x^{2}\right\rangle_{0} r_{k}}{\left(4 r_{k}^{2}+\omega^{2}\right)^{1 / 2}}$,

and

$\bar{\phi}(D)=\arctan \left(\frac{\omega}{2 r_{k}}\right)$

with $\left\langle x^{2}\right\rangle_{0}$ the variance of the stationary unperturbed process. The spectral power amplification (SPA) [43, 44], defined as the ratio of the power of the driven oscillation to the power of the driving signal at the driving frequency $\omega$, is obtained as:

$$
\begin{aligned}
\text { SPA } & =\left[\frac{\bar{x}(D)}{A_{0}}\right]^{2} \\
& =\left[\left(\frac{\mu-3 v}{2 \mu}\right) \beta_{\mu \nu}\left\langle x^{2}\right\rangle_{0}\right]^{2} \frac{4 r_{k}^{2}}{\left(4 r_{k}^{2}+\omega^{2}\right)}
\end{aligned}
$$

Using the theory of SR [3] and looking at the problem in terms of output signal power spectrum, the phase-averaged power spectral density is given by $[3,43]$ : 


$$
\begin{aligned}
S(\Omega)= & (\pi / 2) \bar{x}(D)^{2}[\delta(\Omega+\omega)+\delta(\Omega-\omega)] \\
& +\left[1-\left(\frac{\mu-3 v}{2 \mu}\right)^{2} \frac{4 A_{0}^{2} \beta_{\mu \nu}^{2}\left\langle x^{2}\right\rangle_{0} r_{k}^{2}}{4 r_{k}^{2}+\omega^{2}}\right] \frac{4 r_{k}^{2}\left\langle x^{2}\right\rangle_{0}}{4 r_{k}^{2}+\omega^{2}} .
\end{aligned}
$$

If we neglect terms of orders higher than $\left(A_{0}\right)^{2}$, the leading term in the expression of the signal-to-noise ratio (SNR) [3] thus reads:

$\mathrm{SNR}=\pi\left[\left(\frac{\mu-3 v}{2 \mu}\right) A_{0} \beta_{\mu \nu}\left\langle x^{2}\right\rangle_{0}\right]^{2} r_{k}+\mathcal{O}\left(\left(A_{0}\right)^{3}\right)$.

For a normal Brownian motion for which $\mu=v$, Eqs. (15), (17) and (19) clearly meet the expressions proposed in the literature [3]. Moreover, from conservation law the work done over a period $\tau=2 \pi / \omega$ of the periodic signal equals the change in internal energy $\Delta E=f(x, \tau)-f(x, 0)$ plus the heat $Q$ absorbed over a period, i.e., $W=\Delta E+Q$. Following Sekimoto's stochastic energetic formalism [45], the work done on the system over a period of the periodic forcing is defined as follows:

$W=-\int_{0}^{\tau} x(t) \frac{\mathrm{d} A(t)}{\mathrm{d} t} \mathrm{~d} t$.

The position $x(t)$ being a stochastic variable it is more accurate to express the work done in terms of its average value. Hence, the average work done over a period is:

$\langle W\rangle=-\int_{0}^{\tau}\langle x(t)\rangle \frac{\mathrm{d} A(t)}{\mathrm{d} t} \mathrm{~d} t$.

With the expressions of $\langle x(t)\rangle$ given by Eq. $(14), \bar{x}(D)$ given by Eq. (15) and $\bar{\phi}(D)$ given by Eq. (16), we find:

$\langle W\rangle=\left(\frac{3 \nu-\mu}{2 \mu}\right) \frac{2 \pi A_{0}^{2} \beta_{\mu \nu}\left\langle x^{2}\right\rangle_{0} \omega r_{k}}{4 r_{k}^{2}+\omega^{2}}$.

The quantity $\langle W\rangle$ given by Eq. (22) also represents the hysteresis loop area (HLA) of the system [46] over a period of the forcing, while Eqs. (17), (19) and (22) predict SR as a function of $D$ with a high dependence on the parameters $\mu$ and $v$ characterizing the diffusion anomaly.

Since we are dealing with a two-state system, the position distribution of the particle is the sum of two delta functions. The variance of position distributions having two delta functions equidistant from the origin is $\left\langle x^{2}\right\rangle_{0}=x_{ \pm}^{2}=1$, for the bistable potential $U(x)$. With this, we find that $S R$ is expected at a noise strength:

$D_{\mathrm{sr}}=\left(\frac{5 v-3 \mu}{4 v}\right) z^{\frac{v-\mu}{\mu}} E_{b}$.
The small noise limit considered, i.e., $0<D_{\text {sr }} \ll E_{b}$, yields the following condition from the noise strength $D_{\mathrm{sr}}$ obtained in Eq. (23):

$\frac{3}{5} \mu<v<\frac{3 Z^{\frac{v-\mu}{\mu}}}{5 Z^{\frac{v-\mu}{\mu}}-4} \mu$.

Setting $Z=1$, the later condition turns to $(3 / 5) \mu<\nu<3 \mu$.

\section{Results and discussions}

In the previous section, we derived the expressions of relevant parameters characterizing SR in bistable systems driven by a periodic forcing, in the regime of anomalous diffusion. In the present section, we shall examine their behaviors with variations of characteristic parameters of the model.

To start, the escape rate $r_{k}$, given by Eq. (8), is plotted in Fig. 1 as a function of the noise strength $D$ in the two distinct diffusion regimes, namely the superdiffusion regime (a) and the subdiffusion regime (b), for some values of the ratio $q=\nu / \mu$.

As one can notice, the escape rate increases with the noise strength following a power law for any value of the ratio $q$. However, for a fixed noise strength $D$, the escape rate will drop with $q$ in the subdiffusive regime, while in the superdiffusive regime, there is a peak value in the escape rate for increasing $q$. The behavior of the escape rate with varying $q$ is more telling via the curves of Fig. 2, where $r_{k}$ is plotted over a broad range of values of $q$ for some values of $D$.

From Figs. 1 and 2, it transpires that only in subdiffusive systems there exists a critical noise strength $D_{c}$, under which the particles will always be confined in one potential well. The existence of such critical noise strength can be derived from the relation $\beta_{\mu \nu}^{-1}=(\nu / \mu-1) E_{b}$ and finds its explanation in a cutoff of the stationary solution Eq. (3) restricting the attainable space [35]. The dependence of $D_{c}$ on parameters characterizing the diffusion anomaly, i.e., $v$ and $\mu$, is illustrated in Fig. 3 .

In Figs. 4, 5 and 6, respectively, we plotted the noise dependence of the spectral amplification, the average work and the signal-to-noise ratio, for some values of the ratio $q$. Here, also we distinguished the superdiffusion regime from the subdiffusion regime in the graphical representations.

The SPA and average work both present a resonantlike behavior for all values of $v$ and $\mu$, and qualitatively exhibit the same behaviors with variation of the noise strength. Indeed both show a decrease in their peak value and a rise of the resonance noise strength with the ratio $q$, for the selected range of values of $v$ and $\mu$, in 

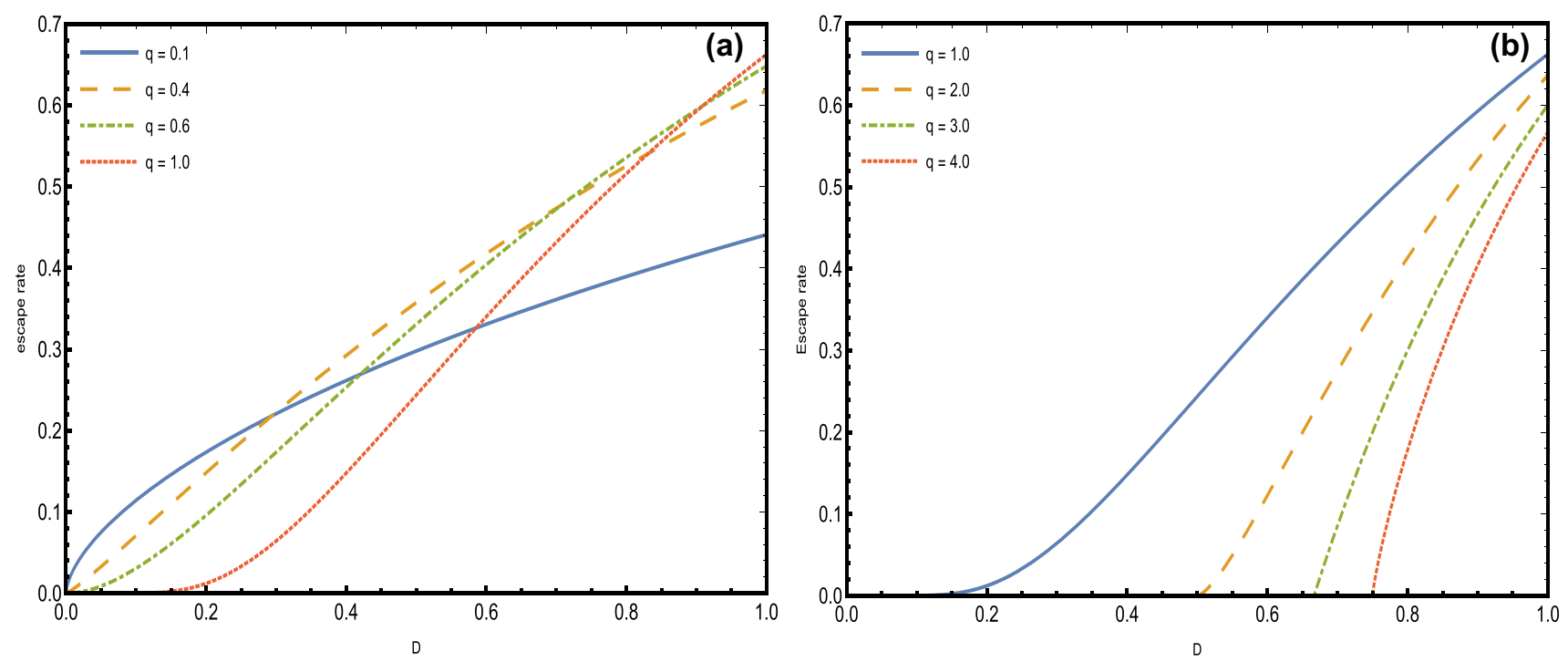

Fig. 1 (Color online) Escape rate $r_{k}$ obtained from Eq. (8) as a function of $D$ for various values of the ratio $q=\nu / \mu>0$ : a superdiffusive system and $\mathbf{b}$ subdiffusive system

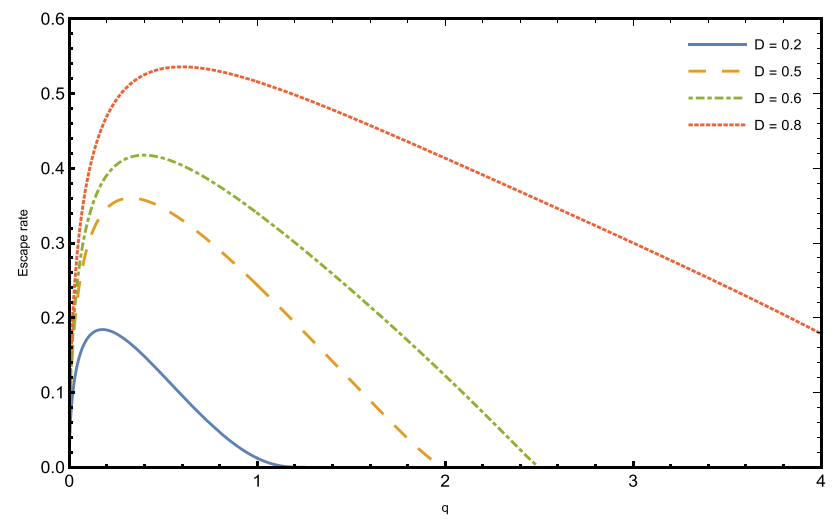

Fig. 2 (Color online) Escape rate as a function of $q$ for some values of $D$

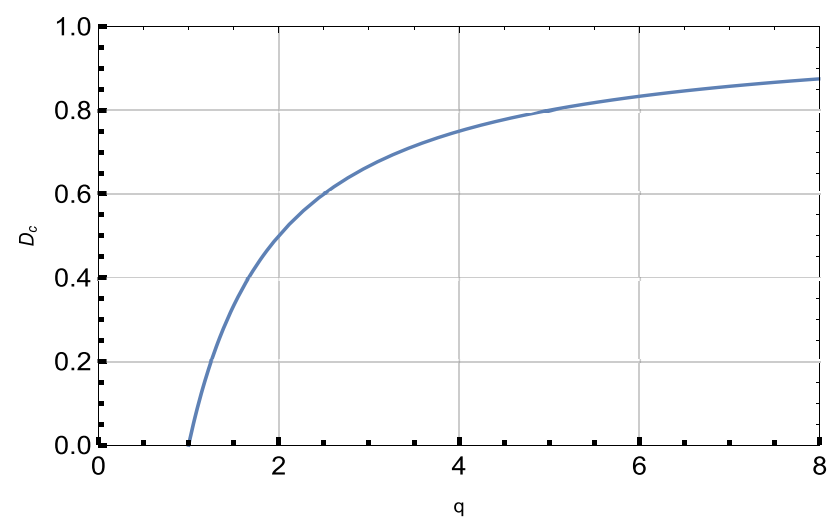

either subdiffusive or superdiffusive regime. The cutoff noise is also reflected in the behavior of SPA and work in the superdiffusive systems, as it restricts the range of allowed noise strengths for the occurrence of SR. The behavior shown by the later quantifiers is also exhibited by the SNR, as shown in Fig. 6 . However, the peak value of the SNR reaches its minimum for $q=1(\mu=v)$ and then rises as $q$ increases. It is also remarkable from curves in Fig. 6a and the analytical expression of the SNR that in the superdiffusive regime, there exist a range of values of $q$ setting the system in a configuration where SR can never occur. Similarly in Fig. $6 \mathrm{~b}$, there is a critical value of $q$ in the subdiffusion regime above which any stochastic resonance should not be considered to occur in the small noise limit. In order to have a broader view on the impact of $v$ and $\mu$ on the three quantifiers, in Fig. 7 we plotted the SPA, the SNR and the work as functions of $q$ considering some fixed values of $D$. From the figure, we remark that at a fixed $D$ the quantifiers strongly diverge in the limit $q \rightarrow 0$, and are nullified for a specific configuration $q_{s}$. From their expressions in Eqs. (17), (19) and (22), this configuration is found to verify the relation $3 v=\mu$. For all values of $D$, the quantifiers will all attain a peak value and then drastically drop to zero as $q$ increases beyond $q_{\mathrm{s}}$. This drop can find its explanation in the fact that the increase of $q$ in the subdiffusive range set the system in a configuration where the noise strength can no more induce transitions between the degenerate stable states.

Fig. 3 (Color online) Critical noise strength $D_{\mathrm{c}}$ as a function of $q$ 

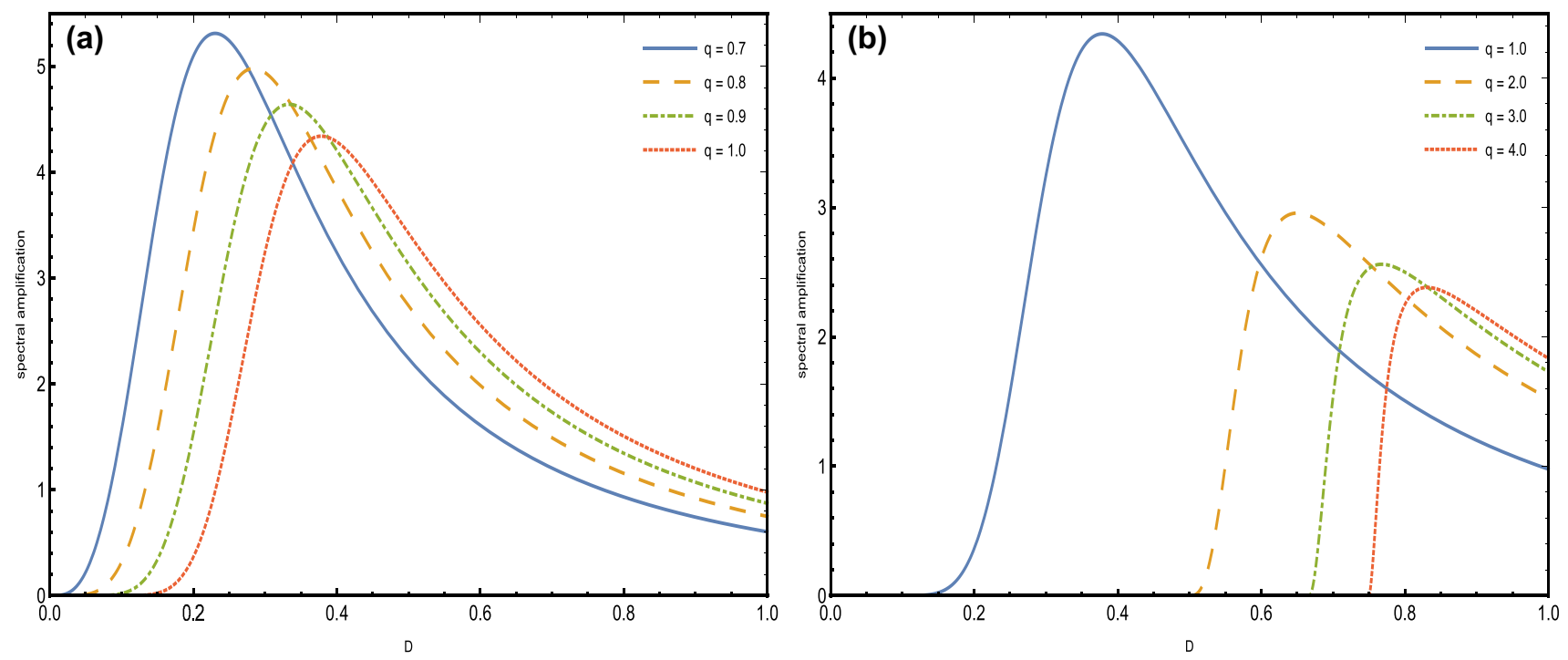

Fig. 4 (Color online) Spectral amplification versus $D$. a Superdiffusion and $\mathbf{b}$ subdiffusion
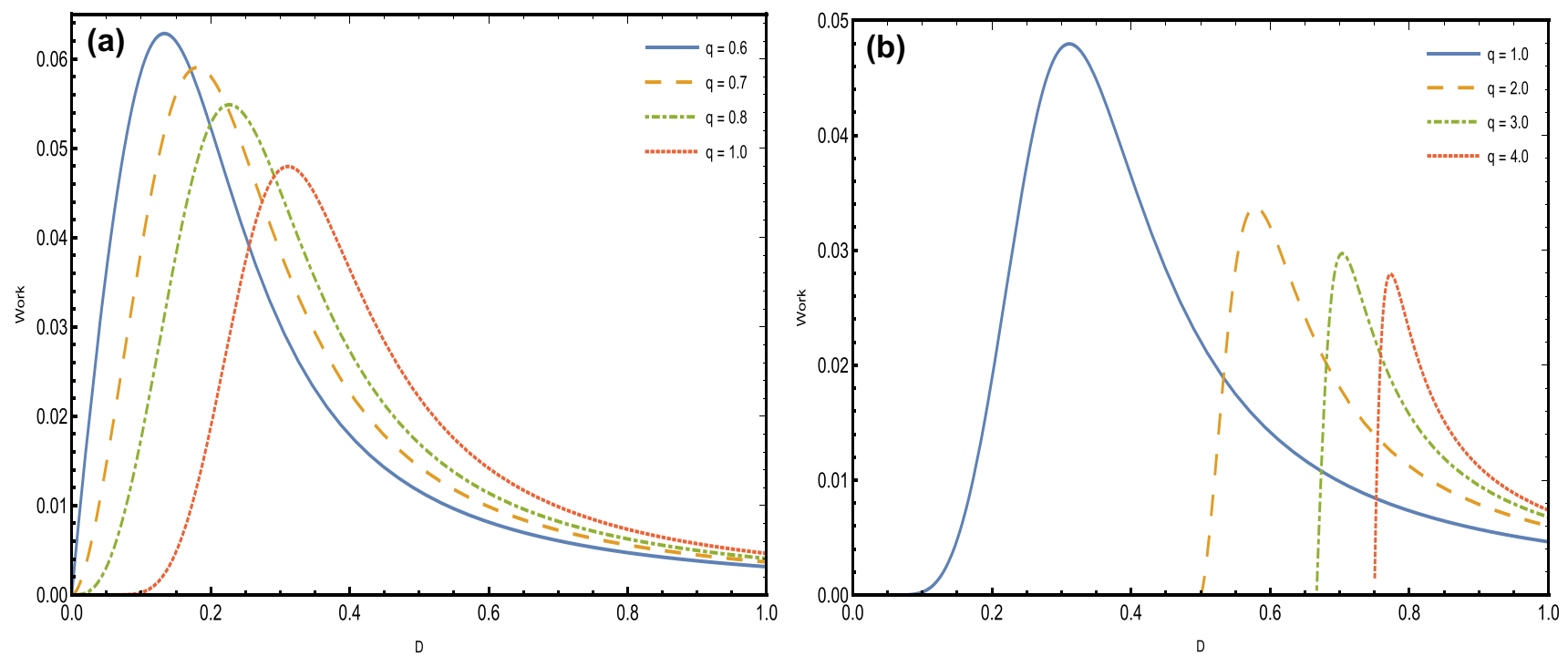

Fig. 5 (Color online) Average work versus D. a Superdiffusion and $\mathbf{b}$ subdiffusion

\section{Conclusion}

We have studied the phenomenon of SR for bistable systems driven by a sinusoidal field and subject to an anomalous diffusion represented by two real parameters, i.e., $v$ and $\mu$. The model describes noise-driven transport phenomena involving either superdiffusive processes when $\mu>v$, normal diffusion when $\mu=v$ or subdiffusive processes when $\mu<v[25,26]$. We predicted SR analytically via a set of quantifiers, including the spectral amplification, the average work done per cycle of the drive force and the signal-to-noise ratio. The occurrence of SR was found to be strongly affected by the order of anomaly of the system diffusion; furthermore, there is a configuration of the 
(a)

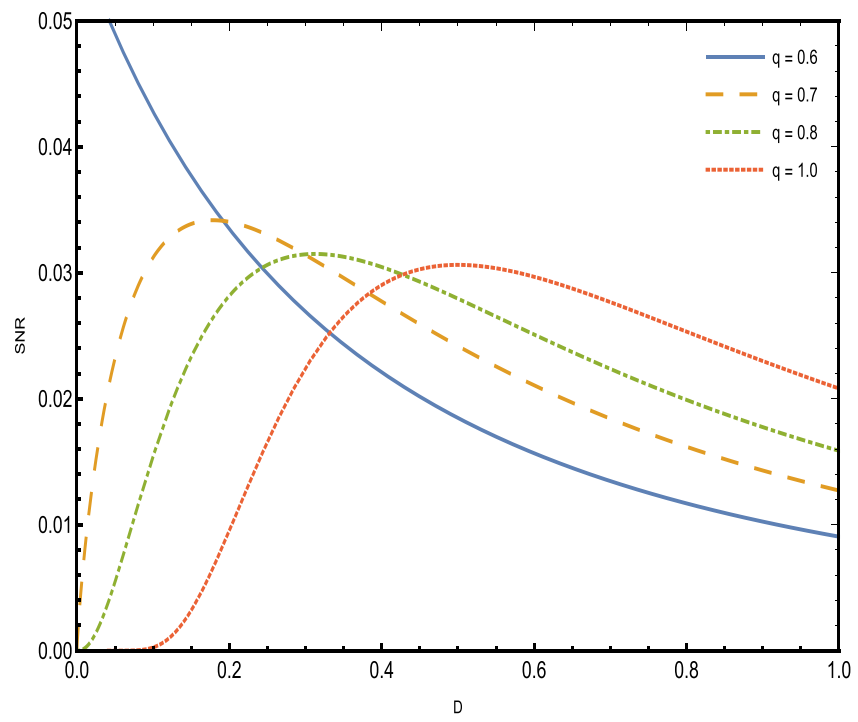

(b)

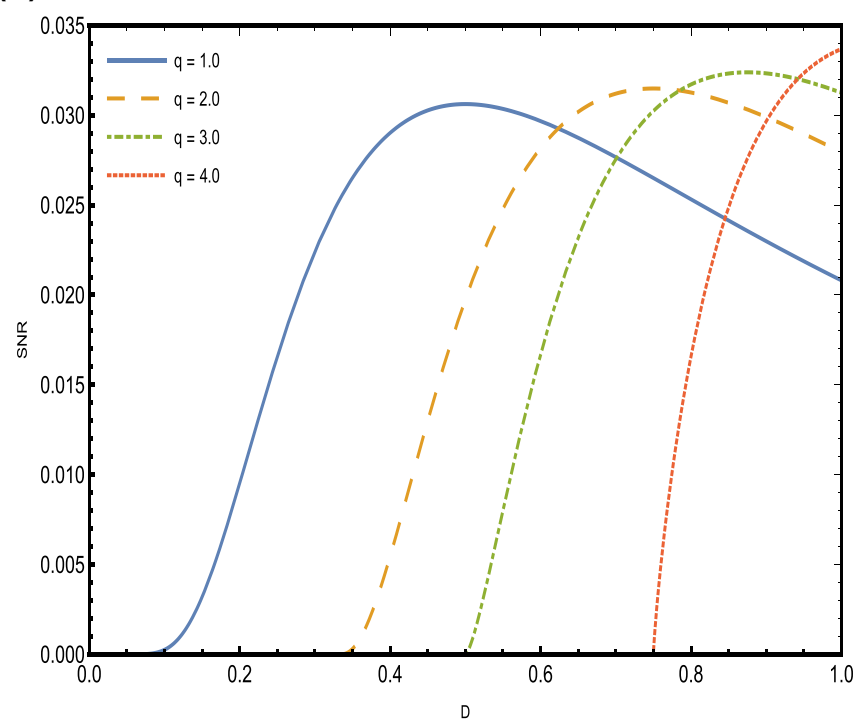

Fig. 6 (Color online) Signal-to-noise ratio versus D. a Superdiffusion and $\mathbf{b}$ subdiffusion

system where the signal-to-noise ratio never shows SR for anomalous diffusion parameters in the range $3 \mu<5 v$. It is important to point out that the peak positions of the three quantifiers were found to be crucially dependent on the order of diffusion anomaly, within the range of validity of the small noise approximation. Our results establish for the first time, the relevant fact that, taking into consideration the interactions of anomalous diffusive systems with a periodic signal, can provide a better understanding of the physics of stochastic resonance in bistable systems driven by periodic forces.
A possible extension of the present study would be to look at the effects of deformability of the bistable potential, such as the change of confinement of the potential well (as done in ref. [22]), or of positions of the doubledegenerate potential minima [14], on profiles of the stochastic resonance quantifiers. Indeed the bistable potential $U(x)$ considered in the present work is the so-called $\phi^{4}$ [11-13] whose rigid profile, reflected in its fixed minima positions and the fixed barrier height, limit their applicability to systems with soft profiles as it is common in polymers and biophysical systems. An analysis of conditions for the occurrence of stochastic resonance in bistable systems with deformable double-well shapes [14] should provide novel insight into the physics of systems of this specific class from a general standpoint. 


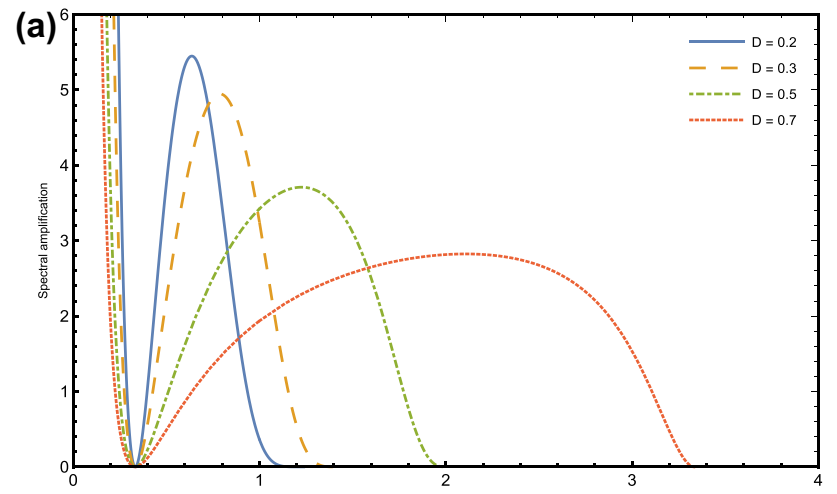

(b)

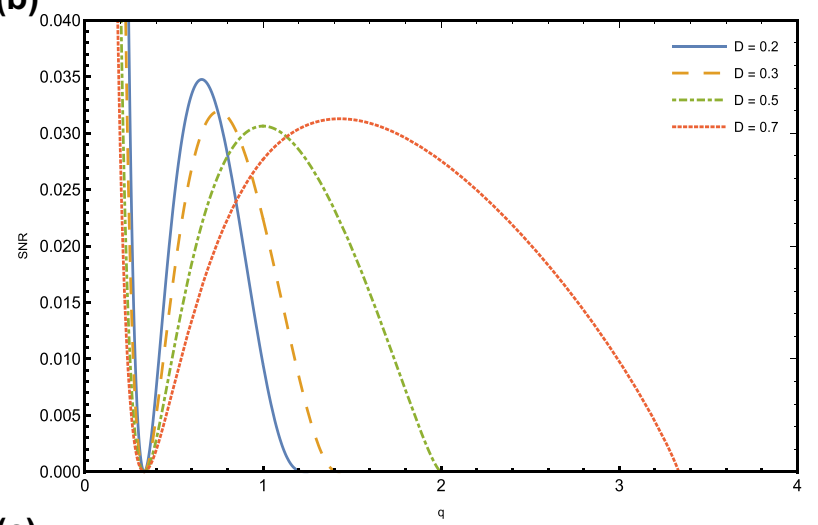

(c)

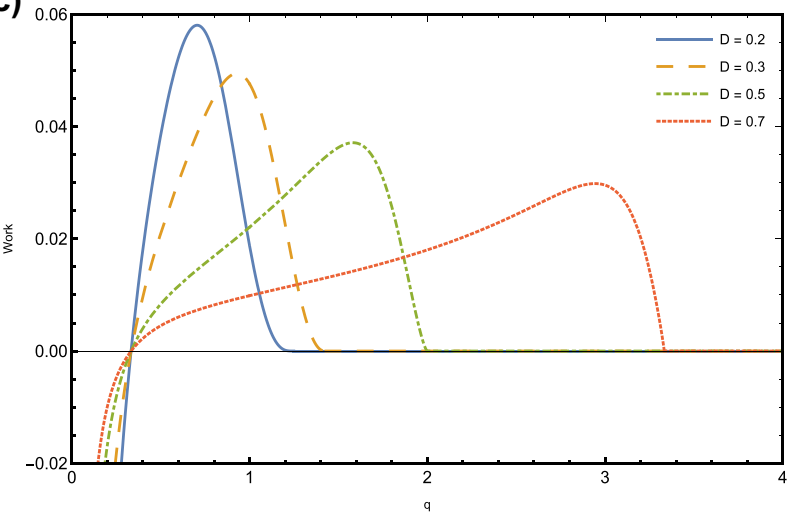

Fig. 7 (Color online) Plots of the quantifiers as function of $q$ : a Spectral amplification, $\mathbf{b}$ signal-to-noise ratio and $\mathbf{c}$ average work

Acknowledgements The second author thanks the Ministry of Higher Education of Cameroon (MINESUP) for providing assistance within the "Research Modernization Allowance."

\section{Declarations}

Conflict of interest The authors declare that they have no conflict of interest.

Open Access This article is licensed under a Creative Commons Attribution 4.0 International License, which permits use, sharing, adaptation, distribution and reproduction in any medium or format, as long as you give appropriate credit to the original author(s) and the source, provide a link to the Creative Commons licence, and indicate if changes were made. The images or other third party material in this article are included in the article's Creative Commons licence, unless indicated otherwise in a credit line to the material. If material is not included in the article's Creative Commons licence and your intended use is not permitted by statutory regulation or exceeds the permitted use, you will need to obtain permission directly from the copyright holder. To view a copy of this licence, visit http://creativecommons .org/licenses/by/4.0\%.

\section{References}

1. Fleming G, Hänggi P (1993) Activated barrier crossing: applications in physics. Chemistry and biology. World Scientific, Singapore

2. Hänggi P, Talkner P, Borkovec M (1990) Reaction-rate theory: fifty years after Kramers. Rev Mod Phys 62:251

3. Gammaitoni L, Hänggi P, Jung P, Marchesoni F (1998) Stochastic resonance. Rev Mod Phys 70:223

4. Wiesenfeld K, Moss F (1995) Stochastic resonance and the benefits of noise: from ice ages to crayfish and SQUIDs. Nature 373:33

5. Risken H (1934) The Fokker-Planck equation: methods of solution and applications, 2nd edn. Springer, Berlin

6. Sutera A, Benzi R, Vulpiani A (1981) The mechanism of stochastic resonance. J Phys Phys A Math Gen 14:L453

7. Nicolis C, Nicolis G (1981) Stochastic aspects of climatic transitions-Additive fluctuations. Tellus 33:225

8. Kim GH, Kang DH, Shin MC (2011) Quantum-classical crossover of the escape rate in the biaxial nanomagnets with a higher order symmetry. Eur Phys J B 83:63

9. Bergues-Pupo AE, Bergues JM, Falo F, Fiasconaro A (2015) Thermal and inertial resonances in DNA unzipping. Eur Phys J E 38:41

10. Geiseler A, Hänggi P, Schmid G (2016) Kramers escape of a self-propelled particle. Eur Phys J B 89:175

11. Krumhansl JA, Schrieffer JR (1975) Dynamics and statistical mechanics of a one-dimensional model Hamiltonian for structural phase transitions. Phys Rev B 11:3535

12. Dikandé AM, Kofané TC (1995) Nonlinear dynamics, soft-mode and central-peak phenomena in one-dimensional systems with long-range interparticle interactions. Physica D 83:450

13. Dikandé AM, Kofané TC (1995) Nonlinear dynamics and thermodynamics of two-component scalar field systems at low temperatures. Physica A 215:104

14. Dikandé AM, Kofané TC (1991) Exact kink solutions in a new non-linear hyperbolic double-well potential. J Phys Condens Matt 3:L5203

15. Berdichevsky V, Gitterman M (1999) Stochastic resonance in linear systems subject to multiplicative and additive noise. Phys Rev E 60:1494

16. Jia Y, Yu S, Li J (2000) Stochastic resonance in a bistable system subject to multiplicative and additive noise. Phys Rev E 62:1869

17. Jia $Y$, Zheng $X, \mathrm{Hu} X$, Li J (2001) Effects of colored noise on stochastic resonance in a bistable system subject to multiplicative and additive noise. Phys Rev E 63:031107

18. Hänggi P, Inchiosa ME, Fogliatti D, Bulsara AR (2000) Nonlinear stochastic resonance: The saga of anomalous output-input gain. Phys Rev E 62:6155

19. Qiao Z, Lei Y, Lin J, Niu S (2016) Stochastic resonance subject to multiplicative and additive noise: the influence of potential asymmetries. Phys Rev E 94:052214

\section{SN Applied Sciences}


20. McNamara B, Wiesenfeld K (1989) Theory of stochastic resonance. Phys Rev A 39:4854

21. Heinsalu E, Patriarca M, Marchesoni F (2009) Stochastic resonance in bistable confining potentials. Eur Phys J B 69:9

22. Rana S, Lahiri S, Jayannavar AM (2011) The role of soft versus hard bistable systems on stochastic resonance using average cycle energy as a quantifier. Eur Phys J B 84:323

23. Sokolov IM (2005) From diffusion to anomalous diffusion: a century after Einstein's Brownian motion. Chaos 15:026103

24. Dhakal S, Sureshkumar R (2017) Anomalous diffusion and stress relaxation in surfactant micelles. Phys Rev E 96:012605

25. Neusius T, Sokolov IM, Smith JC (2009) Subdiffusion in timeaveraged, confined random walks. Phys Rev E 80:011109

26. Li C, Zhao Z, Chen Y (2011) Numerical approximation of nonlinear fractional differential equations with subdiffusion and superdiffusion. Comput Math Appl 62:855

27. Nepomnyashchy AA (2016) Mathematical modelling of subdiffusion-reaction systems. Mat Model Nat Phenom 11:26

28. Lacasta AM, Sancho JM, Romero AH, Sokolov IM, Lindenberg $\mathrm{K}$ (2004) From subdiffusion to superdiffusion of particles on solid surfaces. Phys Rev E 70:051104

29. Weiss M, Elsner M, Kartberg F, Nilsson T (2004) Anomalous subdiffusion is a measure for cytoplasmic crowding in living cells. Biophys J 87:3518

30. Larsen EW, Pomraning GC (1980) Asymptotic analysis of nonlinear Marshak waves. J Appl Math 39:201

31. Gurtin ME, MacCamy RC (1977) On the diffusion of biological populations. Math Biosci 33:35

32. Spohn H (1980) Kinetic equations from Hamiltonian dynamics: Markovian limits. Rev Mod Phys 52:569

33. Buckmaster J (1977) Viscous sheets advancing over dry beds. J Fluid Mech 81(1977):735-756
34. Ploubarinova-Kochina PY (1962) Theory of ground water movement. Princeton University Press, Princeton

35. Jianglin Z, Dong J, Wenping G (2006) Kramers escape rate in nonlinear diffusive media. J Chem Phys 124:024112

36. Lenzi EK, Anteneodo G, Borland L (2001) Escape time in anomalous diffusive media. Phys Rev E 63:051109

37. Tsallis C, Buckman DJ (1996) Anomalous diffusion in the presence of external forces: exact time-dependent solutions and their thermostatistical basis. Phys Rev E 54:R2197

38. Vilar JMG, Rubii JM (1996) Divergent signal-to-noise ratio and stochastic resonance in monostable systems. Phys Rev Lett 77:2863

39. Borland $L$ (1998) Ito-Langevin equations within generalized thermostatistics. Phys Lett A 245:67

40. Pick S (1980) Comment on the difference between Langevin and Ito theories of stochastic differential equations. Physica A 103:630

41. Franck TD (2005) Nonlinear Fokker-Planck eqautions: fundamentals and applications. Springer, Berlin

42. Borland L (1998) Microscopic dynamics of the nonlinear FokkerPlanck equation: phenomenological model. Phys Rev E 57:6634

43. Herrmann S, Imkeller P, Pavlyukevich I, Peitchmann D (2014) Stochastic resonance: a mathematical approach in the small-noise limit, Rhode Island, AMS, Providence, p 194

44. Imkeller P, Pavlyukevich I (2002) Model reduction and stochastic resonance. Stoch Dyn 02:463

45. Sekimoto K (1997) Kinetic characterization of heat bath and the energetics of thermal ratchet models. J Phys Soc Jpn 66:1234

46. Dybiec B (2009) Lévy noises: double stochastic resonance in a single-well potential. Phys Rev E 80:041111

Publisher's Note Springer Nature remains neutral with regard to jurisdictional claims in published maps and institutional affiliations. 